\title{
A new methodology for the study of Mind-Wandering process
}

Nadia Dario, Ph.D in Educational Science, Ca' Foscari University (Italy) and IBEF - International Centre "Ideas for the Basic Education of the Future" (ECNU, China).

Email: nadia.dario@unive.it Tel. number: 347-6253665

ORCID: 0000-0002-1504-3025

Luca Tateo, Federal University of Bahia, (Brazil) and IBEF - International Centre "Ideas for the Basic Education of the Future" (ECNU, China).

ORCID: 0000-0002-3207-6312

\begin{abstract}
Mind-wandering is a quite common psychological experience that raises a number of issues, when occurring for instance at school. Is it a productive or disruptive phenomenon? The current research focuses on pre-post methods that lose the essential of the process. The article proposes a new methodological approach to observe mind-wandering in its development. Italian students of different school grades have been observed and interviewed using a new protocol in order to approach the phenomenon, according to an ecological valid context approach, giving importance to the array of situations and interviewed students (individually or in group) on their mind wandering experience. The analysis shows that mind-wandering con be understood as psychic process rather than an attention deficit or a compensation for boredom. It is a complex mental experience in which several aspects of body-mind and social interaction converge into elaborated meaning-making processes.
\end{abstract}

Keywords: mind-wandering, ecological observation protocol, cultural psychology of education, qualitative methods. 


\section{A new methodology for the study of mind-wandering process}

\section{Introduction}

Mind-wandering is a well-known, fascinating yet not completely understood phenomenon of human psyche. It happens about 4000 times a day with a minimum duration of 14 seconds and it is usually considered a negative event. It is particularly undesirable when it happens at school (Beaty, Benedek, Silvia \& Schacter, 2016; Wammes, Boucher, Seli, Cheyne \& Smilek, 2016). The literature refers to it in different ways: mind-wandering (Mason et al., 2007; Smallwood \& Schooler, 2006, 2007); daydreaming (Singer, 1966); or absentmindedness (Cheyne, Careers \& Smilek, 2006) that "may interfere with educational and occupational performance" (Robertson, Manly, Andrade, Baddeley \& Yiend, 1997; p. 748). In general terms, mind wandering is a process in which attention moves from the here and now, and from a specific task to explore mainly internally self-generated contents (Smallwood \& Schooler, 2015).

"We all experience our minds drifting away from a task toward unrelated inner thoughts, fantasies, feelings, and other musings [...] a shift in attention away from a primary task toward internal information, such as memories [...] Controlled processing is generally associated with the intentional pursuit of a goal. Mind wandering, however, often occurs without intention [...] Thus, mind wandering may entail situations in which individuals temporarily fail to notice that their immediate goal of task completion has been temporarily displaced by another concern" (Smallwood \& Schooler, 2006; p. 946).

The lack of attention, for instance in classroom, is generally considered a waste of time, an absence of commitment and interest, and a lack of participation. Mind-wandering is usually considered both a sign and a cause of potential school problems (Epstein,2011; Xu, 2019; Fray \& Gore, 2018; Di Pietro et al. 2001; (Unsworth \& Mc Millan, 2017; ), with a supposed serious impact on academic performance. Mind-wandering can be the consequence of both minor and major accidents (Broadbent, Cooper, FitzGerald, \& Parkes, 1982; Reason \& Mycielska, 1982), and can indicate 
professional (Reason, 1990), and educational difficulties (Brown, 1927; Lindquist \& Ma Lean, 2011).

However, the existing research on such a relevant psychological phenomenon is still very open and provides several distinct interpretations. Maybe, the advancement in the understanding of mindwandering (MW) requires a different methodological approach. The current methods are based on normative perspective, that considers MW a waste of time, an interference in thinking or something to be prevented or blocked. MW can present itself in different forms: cognitive failures, resting state, distraction, attentional failures, absent-mindedness, issues of concentration on environmental events, and fantasies ${ }^{1}$. Sometimes, the person is unable to provide a full report of her daydreams and has a low awareness of what is happening to her. In other cases, it involves poorly expressive emotions and rumination by the "daydreamer". In a third form, MW implies a positive and constructive mental journey, openness to experience and exploration of ideas, imagination, feelings and sensations. According to Seli, Risko, Smilek \& Schacter (2016), mind-wandering can also occur with or without intentionality.

All the studies about mind-wandering are characterized by a pre-post design, even the most recent attempts (Pepin, et al., 2018) do not deviate very much from the idea that mind-wandering is a distraction from the task. The theoretical and methodological proposal in this article is to study mind-wandering as a developmental process, through an observational protocol, based on complementary perspectives such as cultural psychology and socio-constructivism (Anderson, 1989; Angrostino, 2007; Valsiner, 2017).

\section{Developmental approach to methodology}

\footnotetext{
${ }^{1}$ Mind-wandering can evoke the family of imaginative processes (Brown \& Tateo, 2018), although the research in this sense is not enough developed. For some of its observed features, mind-wandering can show some elements of fantasy and imagination (Harris, 200; Zittoun \& Cerchia, 2013). However, for the lack of satisfying theoretical and empirical research, we will not discuss the relationship between these phenomena, because it deserves a specific treatment outside the scope of this paper.
} 
Human beings are open systems in exchange with the environment, operating in connection to social and relational expectations and temporal dynamics. The school classroom is an example of social and cultural context, in which the processes of meaning construction must be observed through developmental methods. Many approaches to development and learning are that they focus on the differences between an initial state " $\mathrm{A}$ " and a final state "B", focusing on the moment before and after the phenomenon takes place. They inform methods who are "blind to the study of development by eliminating developmental phenomena at the outset - through treating the fuzzy 'borders zones' between A and B as an 'error'” (Valsiner \& van der Veer, 2014, p. 152).

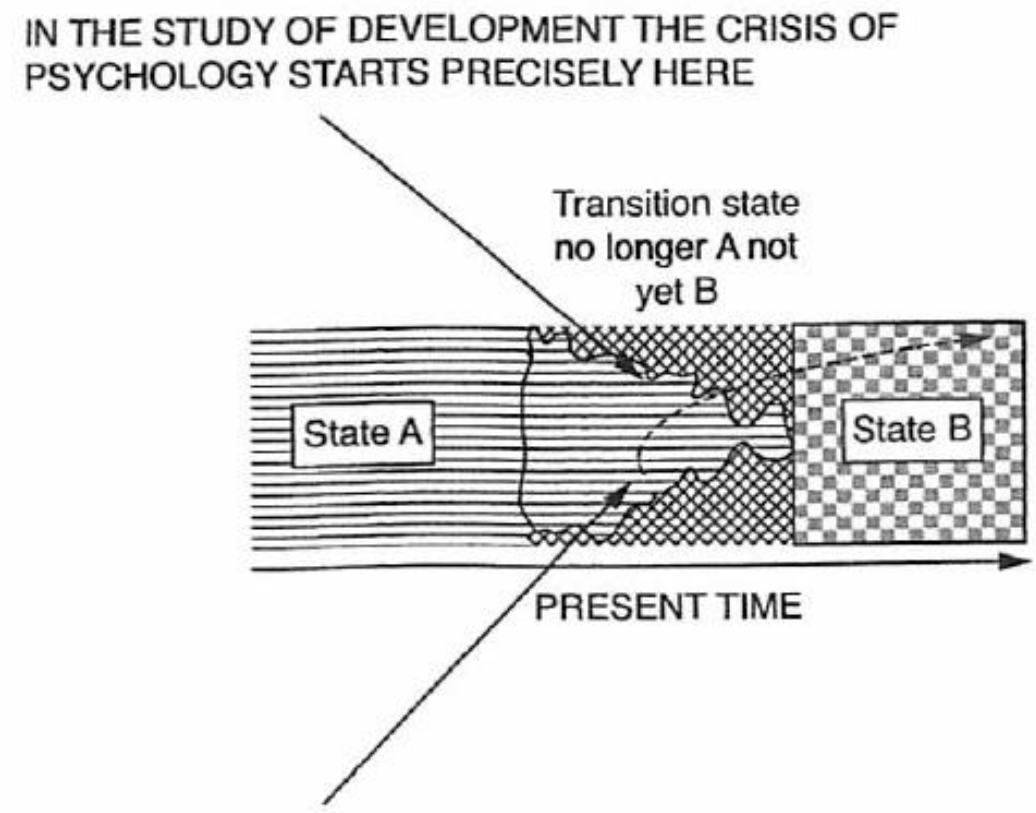

The EDUCATION EFFORT work precisely on the locus of transition

Figure 1 the locus of development and education (Valsiner \& van der Veer, 2014, p. 152).

The current "ecological" methodologies, such as the probe-caught method (Smallwood \& Schooler, 2006) or triangulation method (Pepin et al., 2018), are still focused on the difference between state "A" and state "B" (figure 1), based on the idea that mind-wandering is an interference with regular cognitive activities, such as the retention of information acquired during the (sometimes boring) lectures. Thus, the methods observe the phenomenon when it is already transformed into something 
else, when the mind-wandering process is over and a recovery cognitive strategy is already in place. The common way of assessing involves periodically interrupting individuals (e.g. with an acoustic signal) while they perform a task, and asking them to report the extent to which their attention was related to on-task or no task-related concerns (Mrazek, Smallwood, Franklin, Baird, Chin \& Schooler,2012a; Pepin et al., 2018; Bastian, Lerique, Adam, Fraklin, Schooler \& Sackur, 2017).

In this article, the innovation proposed is to overturn the perspective and try to access the content of mind-wandering as the process unfolds, accounting for the student's first-person perspective in combination with the researcher's observation (Schraube \& Højholt, 2015). The first-person perspective, as Schraube (2013) describes, is based on an fundamental epistemological assumption:

"If we look at human subjectivity more closely, it becomes apparent that its psychological dimensions, such as experience, emotionality, thought and action, are present in a specific form of existence - the first-person mode. On the one hand human experiencing, feeling, thinking, acting with and in the world are always sociomaterially mediated processes (through language, others and the social and technological world); on the other hand they are always someone's processes.

They exist only from the point of view of a subject that has them, and in this sense they always exist in a perspectival mode and therefore in the first-person perspective. This insight is based on the distinction between ontological subjectivity and objectivity They exist only from the point of view of a subject that has them, and in this sense they always exist in a perspectival mode and therefore in the first-person perspective. This insight is based on the distinction between ontological subjectivity and objectivity" (Schraube, 2013, pp. 20-21) 
It is methodologically very difficult to access of mind-wandering while it is occurring, because it would imply a form of interference by the researcher. However, we think it is not necessary to interrupt the MW experience to study it. We try to access the MW indirectly, through a method that combines different temporal and personal perspectives on the vent, shortly after takes place.

The methodology will be presented in the context of a study about students' experience of mindwandering in the classroom of different grades and learning-teaching situations. Each classroom activity is reviewed in the immediate aftermath with the participants, who give their interpretation of their mind-wandering (Stigler, Gallimore \& Hiebert, 2000). Through student-centered perspective, the study examines the conditions in which MW is more likely to emerge, its topical content (phenomenology, sensorial effects, etc.), temporal organization (past experience, routines, etc.), and orientation toward future action.

\section{Mind-wandering in classroom}

Creating, focalizing and maintaining attention in classroom is always a major issue in teaching and learning. The art of teaching and learning is in a certain sense the ability to enter the flow of frequent alternations between focused attention and intentional or unintentional mind-wandering, a situation that is nowadays even more complicated, for instance, by the use of technologies (Aagaard, 2015). Some studies (Smallwood \& Schooler, 2006; Wammes \& Smilek, 2017) have detected that mind-wandering varies in relation to the task's difficulty. It increases during an average lecture, while it remains steady in quiz or test situations. Some studies suggest that mindwandering facilitates the incubation of ideas and correlates with creativity (Smallwood \& Schooler, 2007; Bindler et al. 1999; Baird et al. 2012). The benefits of mind wandering would be future thinking and creative thinking, as it produces anticipation and autobiographical planning.

Despite the number of studies, it is still to understand what is happening during mind-wandering and which factors are at play. The mind seems to "travel" through places and times and to self- 
generate a flow of images, memories, fantasies and inner monologues. Students' mind-wandering is usually considered negatively and it is frequently associated with poor learning, special needs or disruptive behaviour.

Mind-wandering seems to compete for resources and reflect a decoupling of attention from the ongoing task toward the internal train of thought as explained by the Executive Control Failures Personal Concern Model (Mc Vay \& Kane, 2010), the Process-Occurrence Framework and the Decoupling Model (Smallwood \& Schooler, 2006). Impairment in primary task performance is often observed during mind-wandering (McVay \& Kane, 2010; Smallwood \& Schooler, 2006), possibly due to a competition for working memory resources, that would otherwise be directed toward the ongoing task (Smallwood \& Schooler, 2006). Studies help to explain prior findings, claiming that individuals with higher working memory capacity, mind-wander more when engaged in a task with few attentional demands but mind-wander less on tasks with greater attentional demands (Levinson, Smallwood, \& Davidson, 2012) and when individuals report lower levels of concentration (Kane et al., 2007). Mooneyham and Schooler (2013) claim that mind-wandering has disruptive effects in reading comprehension; in test sustained attention; and negative correlation with score of working memory or aptitude tests/measurement. Mind-wandering is also considered a mental activity of scarce utility (Jefferies, Smilek, Eich, \& Enns, 2008; Olivers \& Nieuwenhuis 2005), and it seems to represent a "take-over" of uncontrolled affectivity and thinking.

For instance, Wammes, Seli, Cheyne, Boucher, \& Smilek (2016a; 2016b) have studied the relationship between intentional and unintentional mind-wandering in a group of students over 12week period, finding that intentional mind-wondering is a cognitive strategy adopted by students when they feel demotivated and disengaged during the course of study. Pachai, Acai, LoGiudice \& Kim (2016) suggest the possibility "to reduce mind-wandering at opportune moments and promote learning" (Pachai, et al., 2016, p. 142), in order to keep students' cognitive well-being. An overlooked aspect of MW is the relationship with the future-oriented nature of human action 
(Tanggaard \& Tateo, 2018), and its meaning for the person. The approach of cultural psychology suggests that imaginative and non-imaginative dimensions of thinking are complementary in the meaning-making process, in the course of goal-oriented intentional actions taking place in contexts of collective activities like education (Tanggaard \& Tateo, 2018). For this reason, we have tried to develop a protocol that observes the temporal and contextual conditions in which MW appears, works and disappears during classroom activities and how it can promote/inhibit students' understanding, learning, participation and motivation. The method records the moment in which MW occurs during lesson, and then uses the recording as mediation for narrative reconstruction produced by the student in first person. So, the methodological approach is able to understand the potential role and usefulness of mind-wandering in learning environment. This can foster reflections upon ways of constructing teaching/learning activities that could educate intentional mind-wandering in a productive way for the learner.

\section{Methodology}

This work is an initial presentation of a new qualitative methodology to study mind-wandering, and to describe its different styles and functions, focusing on real life school situations. As a first presentation of the methodology, it is not aimed at assessing its quality criteria (Noble \& Smith, 2015). All useful details about the observation protocol will be provided, so that other researchers can use it and assess its validity, replicability and reliability. Adopting a cultural psychological perspective (Valsiner, 2017), we developed a specific empirical protocol using a mix of methods that would capture both the qualitative and the quantitative attributes of MW situations in the classroom. For this sake, we chose to observe different types of activities in different school grades while most studies in educational field have focused on university students and on a single primary task. 


\section{Participants and Design}

The observations we will discuss are part of a larger study on 117 Italian students of Primary $(\mathrm{N}=36 ; 16$ female), Middle $(\mathrm{N}=42 ; 22$ female) and High School $(\mathrm{N}=25 ; 14$ female), aged between 8 to 18 and 7 teachers have been involved in the study on voluntary basis. The teachers ( 3 from primary school, 2 from middle school and one from high school) participated in a kick-off meeting to discuss the protocol and to complete a questionnaire about the learning activity. Students were divided in three groups corresponding to three learning situations: traditional frontal lecture, testing and cooperative learning. Each participant completed the observational protocol. All the participants completed the observational protocol (117 students were interviewed once, and 5 participants were interviewed twice, both individually and as part of a group, at different times). The study is a first exploration of the new methodology in a natural context. Thus, no selective sampling criteria has been adopted, and the research covered a wide range of learners. In this paper, we will present the results concerning only the participants of secondary school (second and fourth year, age range 14-17), as an example of how the protocol is applied and the data constructed and analysed. For each learning situation, we selected:

- students for individual interviews, according to the number of mind-wandering they experienced during the class activities (reported by the marker balls they dropped, see phase 4 below). Participants were selected according to the explorative goal of the study: those with more MW episodes, those with few and those without any episode reported.

- group of students, who reported MW episodes, were sitting together and showed more reciprocal interactions.

\section{Observation protocol}

A quite simple observation protocol has been constructed, using a mix of observation and interviews. The protocol consisted of: 
- a brief questionnaire, completed by teachers before each lesson in which they stated the contents and the methodologies used.

- a 15/20-minute video-recorded lesson in which the students marked mind wandering by inserting a small cotton ball in a plastic cup. In the current study, we provided students with four cotton balls of different colours.

- a semi-structured interview conducted by researcher after 20/15 minutes. We interviewed students on the mind wandering they had experienced during recorded lesson and also while they were watching it on the computer. The students' interviews were either individual, face to face, or as part of a group.

The protocol is built to include a description of the whole teaching/learning process, and to grasp the perspective of the different actors involved (students, teachers, and researchers). The choice of the semi-structured interview form is based on the need of a script to guide the interview; prepare material to facilitate the recording of the dialogue and systematize the interview in a textual form. This process of constructing texts creates a logical structure for each response that in turn facilitates data analysis. In this regard, the current study has been conducted using semi-structured interview but the researchers prepared the questions in accordance with principles such as: clarity, a series of open and focused questions, avoiding instructions and arranging questions logically (Ergenekon, 2007).

\section{Data Collection protocol:}

The methodological goals of this exploratory study is to develop an observation protocol that is sensitive to both the personal meaning-making of the student and the ecological conditions in which the MW episodes emerge and fade away. We are aware of the tentative nature of the method and the problem of validity and reliability related to a new technique. However, we think it is worth 
detailing the protocol in order to allow other researchers to test it, even in educational context different from a school. The process is composed of the following phases

- Phase 0: Preparation. The researcher establishes a collaboration with the school, meeting the principal and the teacher who is willing to participate to the study. The group identifies the class, answers all parents' questions and acquires their consent. In this phase, the researcher and the teacher begin to discuss the kind of classroom activities that can be included in the study.

- Phase 1: The teacher/instructor is asked to fill in a questionnaire describing their planned teaching/learning activities that will be observed (in Appendix). It is also a way of reflecting on the organization of the time and tasks and to identify potential moments in which MW could emerge.

- Phase 2: The researcher presents the study and the task to the students: they will participate in the classroom activity and will put a cotton ball in the container on their desk every time they realize they are having a MW experience. Then the researcher hands out the balls (a maximum of three balls for video-recorded 20 minutes. If they need more balls, they can indicate it/the numbers on the sheet of paper that they receive at the end of set time) and she will make sure that participants have understood the task they will have to perform. Students can ask questions and receive answers.

- Phase 3: Video-recording of the activity carried out in the ecological context (at school/training institution). We used two different cameras, one recording the whole classroom and one focusing on some groups (small group case). During phase 2, in order to minimize the "camera effect", we had the cameras present but not recording data for the first 15 minutes.

- Phase 4: Immediately after the classroom observation, the researcher watches the videorecording (for about 20 minutes) and identifies single students or groups of students that could be interesting for the interview, according to the research questions. One element of 
interest could be the number of markers (many or no ball at all) put in the container. Other factors of interest for the choice of groups of students, for instance, are the position in the classroom and the numbers of interactions within the groups (Figure 2).

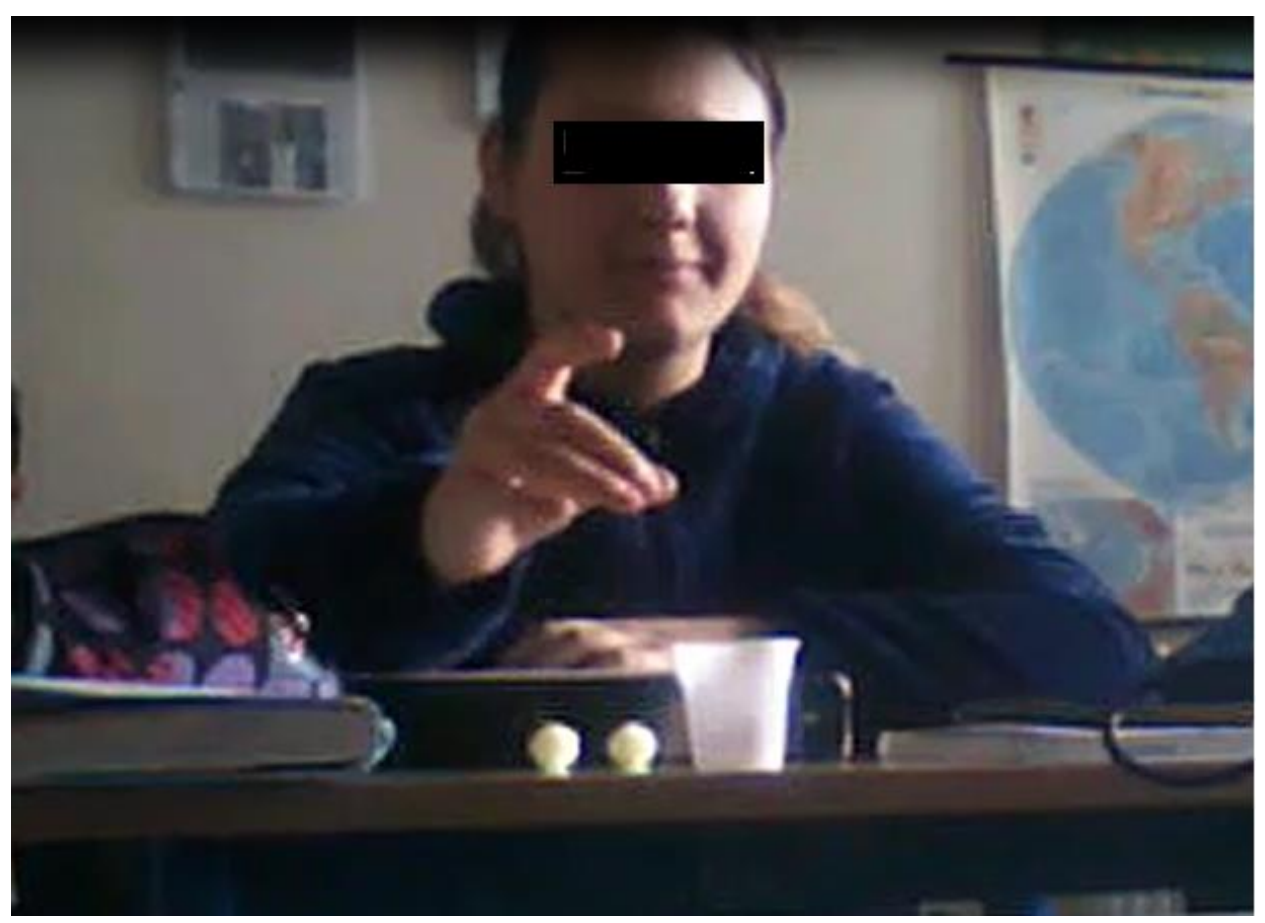

Figure 2 a girl inserting a cotton ball in a plastic cup.

- Phase 5: Students are invited individually (single S) or with other classmates (group G) to an interview with the researcher in a separate room. The researcher explains to the interviewees that the participant's responses will remain anonymous. Each interview (video-recorded) is divided into two part: firstly we ask students about their experience and what they remember about MW episodes. Then, they can watch some of the video-recorded moments of the activity in which they put marbles in the container and are asked about those moments, the transitions in and out MW episodes, the content of the episodes and the modality (visual, imageless, auditory, etc.). The conversation is terminated when we find an answer for each main theme, without a step by step interview. 


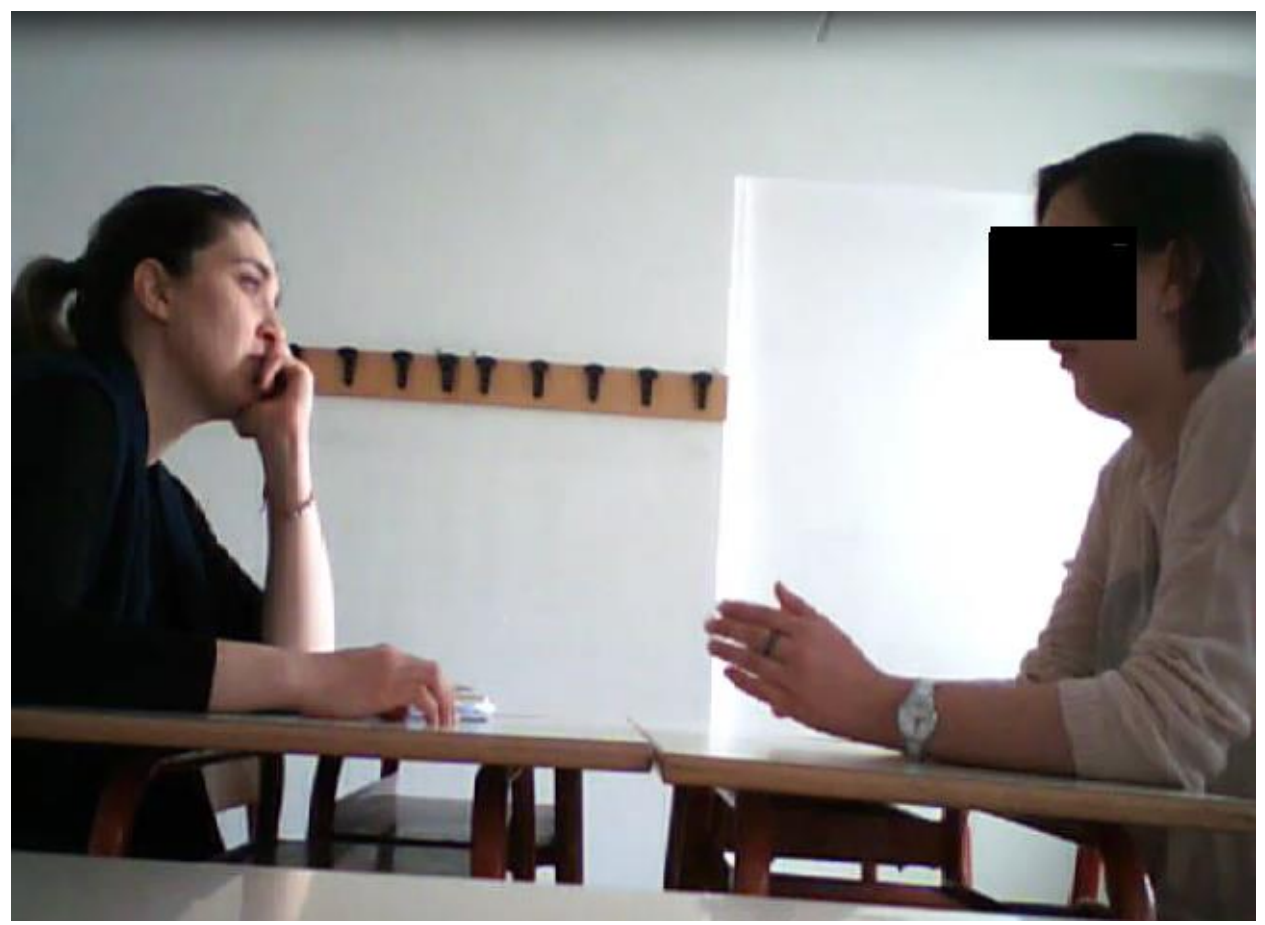

Figure 3 illustrates a single interview case

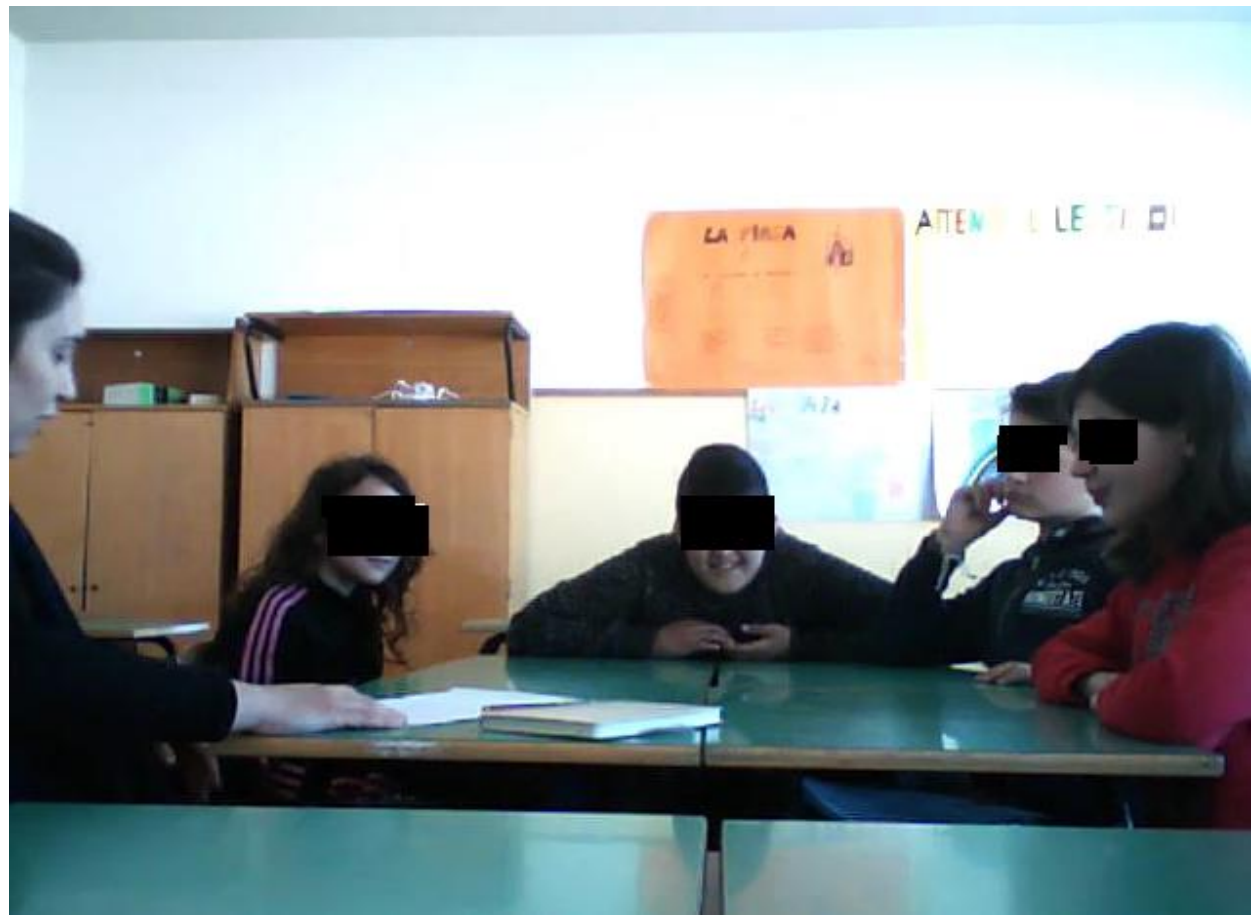

Figure 4 illustrates a group interview case

The participants were chosen on a voluntary basis and on the number of marbles inserted in the plastic cup. The group participants were selected by the interviewer after watching the recorded lesson. In an average class of 22 students, we interviewed $7 / 8$ people for each learning situation 
(Example: After the lecturing, we conducted 7 conversations: 4 single students' interviews of subjects who had inserted 0 marbles, 1 marbles, 2 marbles and three or more marbles; 3 or more students in a group taking into account the interactions had during the video-recorded lesson). Before the classroom activity, the teachers received their questionnaire, and the researcher gave the students an informative message and remained in the classroom to answer questions ${ }^{2}$.

"All you have to do during this lesson, is signal by inserting a marble into a plastic cup when you notice that your mind is beginning to wander, you are not completely focused on the task at hand and / or you are thinking about something else.

Don't worry, there is nothing strange or unusual about all this; in fact, it happens about 4000 times a day. The purpose of the research you are participating in is to understand its usefulness in education. I remind you that the entire procedure is video-recorded and will be commented upon with those of you that will be interviewed “

Afterwards, the researcher viewed the video and then conducted the participants to another room where she gave them an introductory speech and informed them that their opinions would be used for scientific purposes. These interviews had a duration of 30/40 minutes each.

The video-recording of the classroom activity is used as a common object, a mediation tool through which student and researcher construct a shared understanding of the student first-person account. It is not a "real time" access to MW, but it is surely a step forward the observation of the phenomenon in its context.

Some reflections on the protocol's implementation

\footnotetext{
${ }^{2}$ In this study, students asked about privacy policy: how information and videos are treated. All students, without exception, gladly, accepted to participate in the study
} 
The first implementation of the research protocol provided a number of insights and triggered critical reflections about the concrete problems the researcher will experience on the field. The first thing to notice is that mind-wandering is generally valued as a negative phenomenon in educational contexts. So, we suspect that participants have underrepresented the frequency of episodes. This cannot be avoided, as it is part of the school culture. We have tried to adopt some solutions to minimize it. First, an initial briefing phase with both the teachers and the students to explain the sense of the study and to discuss the value of mind-wandering. A concrete example is represented by the choice of the materials for the remembering strategy. The protocol has been playfully called by our participants "the research with coloured ball in the cups". Actually, in the first version of the protocol, we used marbles, but they were too noisy when the students tried to put them into the plastic cup. For the rest of the experiment, we replaced the marbles with coloured cotton balls. Reducing the annoying noises during the lesson made easier for the student to perform the minimal action without being embarrassed to publicly state that he/she was having a mind-wandering episode. Secondly, it made it easier for the participants to recall the mind-wandering during the interview. The balls of different colours mark the space and time of the episode and it gives us the possibility of asking students to remember the ball they want to start from.

The third aspect is the numbers of cotton balls given to the students, three for each person. We did not consider the possibility that students can have more than 3 mind-wandering in 20 minutes. So, we handed a sheet for the registration of mind-wanderings actually occurring in that period of time. Another important reflection concerns the need to make sure that the interview and the interaction in general are a comfortable and pleasant experience and that each participant felt at ease. We noted that the topic of mind-wandering is associated with poor school behaviour and participation. So students are not willing to expose themselves too much, being afraid of being assessed negatively. We had to make every effort to prevent the interview from turning into an interrogation or a school assessment, by forming unbiased and empathetic interactions. This is in general a very relevant 
aspect of empirical working educational contexts, which is not enough stresses in the methodological debate.

Finally, we are aware of the limitations of the protocol with respect to the ecological validity. Our aim is to observe the phenomenon in its temporal making. It would imply having direct access to the experience in its making. Of course, this would create an interference in the MW, as any of the existing study method unfortunately does. In order to minimize the issues of the retrospective reconstruction, we adopt two solutions: first, we interview the participants immediately in the aftermath, few minutes after the end of the activity; second, we use the cotton balls and the video recording as mediation tools to facilitate the student first-person account. It allows to obtain precious information not only about the content of the MW, but also about the initial and final conditions of the experience and the context in which it occurred. In any case, methods of psychological investigation are based on triggering participants' acts of guided introspection (Valsiner, 2017). Any form of externalization and communication of a psychological experience occurs de facto in the aftermath of its making.

\section{Example of data analysis}

In order to complete the description of the methodology, it is worth mentioning the way data are analysed. It is not an exhaustive description, because the information collected with this methodology lends itself well to different methods of analysis. In this paper, we present an interpretive content analysis to understand participants' narrations. The students' answers to each question have been categorized to observe similarities and differences in the content of mindwandering. Data are analysed taking into account the research questions and the conceptual framework of the research and data are qualitative categories. Five main themes are developed as a result of this step (table 1): MWS contents; Activation and engagement before MW; Time frame and direction of MW; Mw's connections with task or relational context; Self-perception during MW; Perception of others during MW; and Sensation of ease while daydreaming. 


\begin{tabular}{|l|r|}
\hline Mw's Contents & $\begin{array}{r}\text { Number in } 202 \\
\text { narrations }\end{array}$ \\
\hline Others $\quad$ Peers & 51 \\
$-\quad$ Relatives & 27 \\
$-\quad$ Others (teachers, lovers, etc..) & 32 \\
$-\quad$ Animals & 6 \\
Objects and Places & \\
$-\quad$ Objects & 54 \\
$-\quad$ Places & 28 \\
Unreal elements & \\
$-\quad$ Unreal & 7 \\
\hline Activation and engagement before MW & $\%$ \\
\hline Tired/bored & 44 \\
Active/hands-on & 32 \\
I don't remember & 24 \\
\hline Time frame and direction of MW & 8,5 \\
\hline Present & 57,5 \\
Future & 28,8 \\
Past & 5,2 \\
Unreal & $\%$ \\
\hline Mw's Connections with task or relational context & 28,3 \\
\hline To the task & 28,4 \\
Not connected to the task & 43,3 \\
Connected to the relational context & $\%$ \\
\hline Self-perception during Mw & 51,3 \\
\hline Yes & 34,8 \\
No & 13,9 \\
\hline Idon't know & 45,4 \\
\hline Perceptions of others during Mw & 43,6 \\
\hline Yes & 11,0 \\
No & 60,6 \\
I don't know & 26,5 \\
\hline Sensation of ease while daydreaming & 12,9 \\
\hline Yes & \\
No & \\
I don't know & \\
\hline
\end{tabular}

Table 1 the 7 main themes and sub-themes emerged through the analysis of the interviews and the absolute and relative frequency of each theme.

\section{Description of the themes}

Theme 1 (MWS Contents), there are three subthemes: others (because it is interesting to consider how interpersonal relations are present in MW), objects and places and unreal elements. The latter ones (unreal elements) remain marginal and associated with primary school children (we pass from imagining disinfecting cotton that comes alive to become a superhero). On the contrary, objects and relatives (the additional subtheme of "others") are described by the majority of students.

Theme 2 (the present of activation/participation and tedium/tiredness) reveals that students are not always totally disengaged from the school lesson but that engagement and disengagement are 
equally divided. This observation seems to confirm that there is no connection between boredom and mind-wandering. Furthermore, in repetitive mind-wandering, the sense of activation and involvement changes (see next paragraph).

The third and fourth themes confirm that mind wandering concerns future planning and it is often connected with self-reflection (Schooler, Smallwood, Christoff, Handy, Reichle \& Sayette, 2011) but it is often connected with relational contests, external stimuli ("I saw the classmate's eyes and I started to think about what she had told me an hour before", "I hear her voice and I remembered that I would be in another State"), limited the idea of spontaneity in mind-wandering.

Theme 5 and 6 present self-perception and perception of others, two elements not considered in previous researches. More than half of those interviewed claim to have a clear perception of their body, of how they are seated and positioned in relation to the teacher and the rest of the class. However, the percentage of students perceiving what the teacher or the group of friends (45\%) was doing is similar to that had no perception $(43 \%)$. The inter-individual variability emerged in this case and mind-wandering appeared as a personal characteristic.

Theme 7 shows that students consider mind-wandering a familiar phenomenon and they are at ease when they daydream (60\% of 202). Therefore, we can conclude that mind-wandering is a repetitive thought but not in itself considered a negative event or experience (rumination).

\section{Interpretative analysis of participants' utterances $^{3}$}

According to Mc Vay \& Kane (2010), Smallwood \& Schooler (2006) Mooneyman et al. (2013), Seli et al. (2014), mind-wandering is a self-generated, non-intentional, off-task thought process in which the individual's own internal thought processes that stray from the goal -relevant thought, in particular in reading comprehension. This is in line with the decoupling model that suggests instances of mind-wandering reflect a decoupling of attention from the ongoing task towards an

\footnotetext{
${ }^{3}$ Participants' opinions were cited by coding them without using their names in accordance with privacy policy. In this regard, individuals were numbered and coded P. Names reported are fictional. In this part, we re-discuss literature on mind-wandering, reporting participants' answers and we also compare these to data analysis.
} 
internal train of thought. On the contrary, MW confirms the existence of an intermediate paradigm: MW is located between two states of consciousness - mental alertness and mental activity during sleep. The interviewees reported that:

"I can have the perception of what's happening, for instance even when the other profs are lecturing...for instance I see the philosophy prof walking back and forth..I know he's doing this but at the same time I am thinking about something else". (P101)

"[...] When it happens, I listen to what the prof is saying, too, and I understand what he's saying but other times I get lost...not this time” (P104)

Such condition of in-betweeness is interestingly related to the body-mind, as reported in the following excerpt:

"114P: Yes, my mate was talking

I: while you were thinking to that stuff?

114P:Yep

I: So you did perceive what was going on around you?

114P:Yep

I: Did you perceive of yourself? Of your own body, of your sitting posture?

114P:No"

The context regulation hypothesis considers mind-wandering an internal thought that could be reduced if high-functioning cognitive system is active and related to the contest. Mw is considered by literature as a way of escaping from present monotonous situation (Wammes, Seli, Cheyne, Boucher \& Smilek, 2016): it is an absent minded job in which students have stopped time; they are lost in another world. But students (P77; P114; P110; P202) seem to have a different understanding of their experiences: 
I: Do you think it happened while you were more tired/bored or more activelengaged?

P77: Buuuf, ...uhm ...don't know...I guess, at least for me, can happen both things..but at that moment...

I: In that case?

P77: ehm..I was active

I: You were active?

P77: Yes, of course, of course

I: Do you remember when that though began?

114P: When I saw the image of all the cars and the broken bridge... and the people over there looking at it.

I: Were you more tired and sleepy or activelengaged, when you had that though?

114P: Active

202P: "There were times when I saw what was happening outside...I started to think and I felt muffled but when I solved (she was referring to a future problem, question)... after everything (the sensation) decreased... Other times...as in this case (second mind-wandering), I heard his voice (classmate) around me...I can't stand his voice!!... and I thought and heard at the same time...but I thought briefly and I was less muffled"(in brackets my interpretations)

Moreover, students reveal a strong connection with previous discussions with their school mates (relational context) and task, showing that the contextual condition play a part in the MW, which id not completely an "escape" from the here and now. 
110P: So, it's not connected (to evening's organization) with what happened to me because my mate said that one hour before and I don't know how it occurred to me!?! I:Ah, ok..it's not connected. When is it occurred to you? 110P: when she looked me for a while.... and I remembered that when she had looked at me, she asked the question (on evening) and I remembered and started to think

114P: On yes, the prof told us about armored car and I remembered a game I play at home ....ehhh...I thought about the armored car and it was a little bit different than other in the video $[\ldots]$

I: Was what you were thinking connected to the task you were doing?

114P:A little but yes

I: A little why?

114P:Beacuse It is linked a little to the car but the topic was completely different.

Mind-wandering seems to be a necessity for all of us: it is a way of limiting tedium (Mooneyman \& Schooler, 2013; Wammes, Seli, Cheyne, Boucher \& Smilek, 2016) especially when the latter occurs frequently. The positive effect of MW is that it is able to regenerate the mind with the result that students end up becoming more alert.

Moreover, this research underlines how emotions activate mind-wandering but we affirm that context is even more powerful. This is how we are able to explain the ambivalence found in many answers.

I: In your opinion, is it an emotion that triggered this thought about bosses and your house? 
111P: Maybe the bosses because Ifelt a bit sad about the things they were saying and then about the house because I was scared ... because today I have to be alone at home and locked in ... so I was a bit scared about that.

I: Do you think there is an emotion that has triggered this thought?

77P: I think so, because I thought of him (sick companion) and, I mean, there was no shyness ... no pain... Compassion. That's it!

This excerpts converge on previous findings: MW works with past, present and planning the future, and simulates potential association with the self; it is connected with creativity (Gruzelier 2014) or autobiographical memory (Baird, Smallwood, \& Schooler,2011; Baird et al., 2012; Smallwood, Ruby, \& Singer, 2013). Our research adds new insight by showing that all of these can also occur during situations in which demands on attention are relatively high. As participants said:

111P: I had an image of the bridge and also the concept, because I imagined how it was when they destroyed it but I wanted to imagine it how it was before they destroyed it..I was thinking it was a bridge like, a highway that like was a kind of bridge beneath, a little bridge with some small..small like I imagined a small river and if you go further, you could see..like areas.. a trail, a side way you can only walk, besides the highway and I was thinking to this while I stopped... (gesturing while explaining) and got back to concentrate on the video.

118P: Then, I have to finish the power-point and when I) finish it...I finish it during the ICT lesson or in the fourth hour with geography. Then I thought asking to the ICT one (teacher), if you would let me finish it and if she says no, I will ask to the geography one)...and in this way, I go to... I mean I produce a mental conversation...It's unavoidable when I try to plan 
something...to have a conversation with its implications...If she says no, I what to say and If she says yes what to ask for. I feel more comfortable, if I solve it during this conversation, I don't get on frustrated.

In the excerpts one can see how the method is able to account for the processual nature of mindwandering. Instead of gathering information about the deviation from the task, one can observe that when students have to solve problems (e.g. being late to prepare a Power-point), there is a mental simulation, an inner dialogue anticipating and planning future courses of action. This can lead to a number of consequences, such as emotion regulation (I feel more comfortable, if I solve it during this conversation, I don't get on frustrated); generativity (I imagined how it was when they destroyed); and reflection (It's unavoidable when I try to plan something...to have a conversation with its implications).

\section{Conclusions}

The study originates from the observation that in the current field of mind-wandering studies there is a need for a methodological approach focused on the developmental and meaning-making processes. The current methods do not grasp the phenomenon in its development, rather focus on its effects on cognitive performance at school. The objective of the present work is thus to present a new methodological approach and a protocol able to explore mind-wandering in a real teachinglearning context and in different school grades. A complementary design has been described, in which the video-recording lessons are complemented with teachers' questionnaires and students' conversations on mind-wandering experience. The study collected narratives on mind-wandering. This allowed to perform and discuss also a potential way of analysing the data obtained with the protocol. We have summarized the mind-wandering experience in seven main themes: contents, activation and engagement before MW, time frame and direction of MW, value of emotion in MW 
activation, self-perception during MW, perception of others during MW, sensation of ease while daydreaming.

The analysis of the interviews allows to introduce several interesting ideas. The mind-wandering contents seems to change according to the interviewees' age because all unreal elements concern children while $62 \%$ of MW are connected with social and relational situations in which peers, teachers or relatives are involved. However, participants produce imaginary simulations, create a virtual scenarios to solve unfamiliar situations, where mind-wandering mind is not necessarily associated with problematic or unhappy conditions. People also use it for effective planning and self-amusement.

The literature generally considers mind-wandering as the inability to keep the attention towards relevant elements, because it is an internal thought not context related. Through the protocol, the contextual elements become more visible, not only in cases in which little attention is required but where also high degree of attention is expected. The specific context (a voice of a classmate, some words connected to a previous discussion, text) plays a key role in the activation and progression of mind-wandering.

When one observes the process in action, a continuum emerges between metacognition and mindwandering. MW can be understood as part of the stream of consciousness (Fossa, 2018). A mental state in which consciousness looks towards inner thoughts, feelings and memories while keeping a connection/link with environmental conditions. According to Immordino-Yang (2016), it is a "constructive internal reflection" that relates to past/future and remembering/planning's continuum. Several students report that during classroom hours they have thoughts concerning their homework. MW is something more complex: it works with factual, counterfactual, hypothetical and with multimodal thought (image, concept, movement, etc.). The protocol allowed us to observe that MW is part of the students' planning activity. They process and re-process the elements collected during a school day, but also from listening to music, watching tv, playing video-games, etc. 
Finally, it is necessary to point out that the conclusions reached by this work should be read in the light of the limits of this research. The main goal of the study was to develop a new methodology to study mind-wandering in its temporal making. The methodology requires further evaluations of its validity and reliability. Some improvements are also required with respect to the number of mindwandering of each student remains unknown, but this data could be implemented in future protocol extension. This data could provide information about the connection between mind-wandering and learning situations. Besides, the research does not explore extensively the importance of emotions in mind-wandering: the interviewees are divided into mind-wandering activated by emotion and not activated by them. However, the methodology has the potentiality to address the problem with the quality of the data obtained.

In conclusion, MW seems to be a complex phenomenon that involves a part of intentionally directed self-generated thoughts, and further research is needed to explore its potentialities (Mooneyham \& Schooler, 2013). Indeed, if MW is a place of creative production and exploration of ideas, plans, scenarios and emotions, it could be potentially educated to become a tool for thinking in a teaching/learning activity. Our methodology can provide the ecological approach to the future research needs to answer some of the main questions:: what are the different forms of mind wandering? The slip-side of thought? Is there a relationship between dreaming and mindwandering? Can educated mind-wandering become a resource for teaching/learning?

\section{References}

Aagaard, J. (2015). Media multitasking, attention, and distraction: a critical discussion. Phenomenology and the Cognitive Sciences, 14(4), 885-896.

Baird, B., Smallwood, J., Mrazek, M. D., Kam, J. W., Franklin, M. S., \& Schooler, J. W. (2012). Inspired by distraction: mind wandering facilitates creative incubation. Psychological science, 23(10), 1117-1122.

Bastian, M., Lerique, S., Adam, V., Franklin, M. S., Schooler, J. W., \& Sackur, J. (2017). Language facilitates introspection: Verbal mind-wandering has privileged access to consciousness. Consciousness and cognition, 49, 86-97. 
Beaty, R. E., Benedek, M., Silvia, P. J., \& Schacter, D. L. (2016). Opinion: Creative Cognition and Brain Network Dynamics. Trends in Cognitive Sciences, 20, 87-95.

Brown, S., \& Tateo, L. (Eds.). (2018). The Method of Imagination. Charlotte, NC: Information Age.

Cheyne, J. A., Carriere, J. S. A., Smilek, D. (2006). "Absent-mindedness: Lapses in conscious awareness and everyday cognitive failures". Consciousness and Cognition 15 (3): 578-592. doi:10.1016/j.concog.2005.11.009. PMID 16427318

Di Pietro M., Bassi E., Filoramo G. (2001). L'alunno Iperattivo. Problemi di comportamento e strategie educative. Erickson, Trento.

Harris, P. L. (2000). Understanding children's worlds. The work of the imagination. Malden, : Blackwell Publishing.

Fossa, P. (2018). The Problem of Reality in Psychology: Revisiting the Reality Concept in Oswald Külpe. Human Arenas, 1(86), pp. 86-96. https://doi.org/10.1007/s42087-018-0002-1

Fray, L., \& Gore, J. (2018). Why people choose teaching: A scoping review of empirical studies, 2007-2016. Teaching and Teacher Education, 75, 153-163.

Immordino-Yang, M.H. (2016). Neuroscienze affettive ed educazione, Milano: Cortina

Kane, M. J., Brown, L. H., McVay, J. C., Silvia, P. J., Myin-Germeys, I., \& Kwapil, T. R. (2007). For whom the mind wanders, and when: An experience-sampling study of working memory and executive control in daily life. Psychological science, 18(7), 614-621.

Levinson, D. B., Smallwood, J., \& Davidson, R. J. (2012). The persistence of thought: evidence for a role of working memory in the maintenance of task-unrelated thinking. Psychological science, 23(4), 375-380.

Mason, M. F., Norton, M. I., Van Horn, J. D., Wegner, D. M., Grafton, S. T., \& Macrae, C. N. (2007). Wandering minds: the default network and stimulus-independent thought. Science, 315(5810), 393-395.

McVay, J. C., \& Kane, M. J. (2010). Does mind wandering reflect executive function or executive failure? Comment on Smallwood and Schooler (2006) and Watkins (2008). 